\title{
GMR
}

\section{Steroid metabolism gene polymorphisms and their implications on breast and ovarian cancer prognosis}

\author{
E.V.W. dos Santos, L.N.R. Alves and I.D. Louro \\ Núcleo de Genética Humana e Molecular Departamento de Ciências Biológicas, \\ Centro de Ciências Humanas e Naturais, Universidade Federal do Espírito Santo, \\ Vitória, ES, Brasil \\ Corresponding author: E.V.W. dos Santos \\ E-mail: eldamariavw@yahoo.com.br \\ Genet. Mol. Res. 16 (3): gmr16039691 \\ Received April 4, 2017 \\ Accepted May 31, 2017 \\ Published July 6, 2017 \\ DOI http://dx.doi.org/10.4238/gmr16039691 \\ Copyright (C) 2017 The Authors. This is an open-access article distributed under the terms of \\ the Creative Commons Attribution ShareAlike (CC BY-SA) 4.0 License.
}

\begin{abstract}
A role for estrogen in the etiology of breast and ovarian cancers has been suggested; therefore, genetic polymorphisms in steroid metabolism genes could be involved in the carcinogenesis of these tumors. We have aimed to investigate the role of GSTP1 and CYP17 polymorphisms and their correlation with MSI (microsatellite instability) and $\mathrm{LOH}$ (loss of heterozygosity) in $A R, E R \beta$ and CYP19 genes in women from Espírito Santo State, Brazil. The study population consisted of 107 female breast and 24 ovarian tumors. GSTP1 and CYP17 polymorphisms were detected by polymerase chain reaction (PCR) amplification followed by restriction fragment length polymorphism (RFLP) analysis while MSI and LOH were analyzed by PCR. GSTP1 and CYP17 polymorphisms alone were not associated with an increased risk for breast or ovarian tumors. However, when combined with MSI/LOH in $A R, E R \beta$ and $C Y P 19$ genes, we were able to detect significant associations with the GSTP1 wild-type genotype in PR (progesterone receptor) negative breast cancers or the CYP17 wild-type genotype in ER (estrogen receptor)
\end{abstract}

Genetics and Molecular Research 16 (3): gmr16039691 
and PR-negative breast tumors. No associations with ovarian tumors were detected. Our results suggest that wild-type GSTP1 or CYP17 genes when combined with LOH/MSI in steroid metabolism genes may play a role in ER and/or PR negative breast cancers. These data support the hypothesis that genes related to steroid metabolism are important in the characterization of breast cancer and that the analysis of single polymorphisms may not be sufficient.

Key words: Breast/ovarian cancer; GSTP1; CYP17; STR markers; Polymorphisms

\section{INTRODUCTION}

Breast cancer is the most common malignancy among females and the most common cause of cancer death among women in Western countries (Imyanitov and Hanson, 2004; Antognelli et al., 2009) and ovarian cancer is the most lethal gynecological malignancy at present (Huan et al., 2008; Lurie et al., 2009).

Germline mutations in the so-called high penetrance genes of breast and ovarian cancer susceptibility, such as $B R C A 1$ and $B R C A 2$, appear to account for the majority of hereditary breast and ovarian cancers, but they represent only 5 to $10 \%$ of breast cancer cases and up to $15 \%$ of ovarian cancers (Lynch et al., 2009; Ramalhinho et al., 2012).

Thus, polymorphisms in low penetrance genes can be linked with a significant percentage of breast and ovarian cancers. Low penetrance genes can be involved in a wide variety of functions including steroid hormone metabolism, detoxification of environmental carcinogens, DNA damage repair genes and tumor suppressor genes (Miyoshi and Noguchi, 2003; Delort et al., 2008; Ramalhinho et al., 2012).

Polymorphisms in the cytochrome P450 family (CYPs) and in the glutathione S-transferase (GSTs) enzymes have been of particular interest because these enzymes play an important role in the metabolism of environmental carcinogens and of estrogen (Torresan et al., 2008; Antognelli et al., 2009), which seems to be involved in breast and ovarian carcinogenesis. Genes of these families are highly polymorphic, presenting alleles with different enzymatic activities and possibly tumor risk (Torresan et al., 2008).

The CYP17 gene encodes the cytochrome P450c17 $\alpha$ enzyme, which is a key enzyme in the estradiol synthesis (Kristensen_and Borresen-Dale, 2000), converting pregnenolone and progesterone to androgen and estrogen precursors (Torresan et al., 2008). The $T \rightarrow C$ polymorphism in the 5 '-untranslated promoter region creates an additional SpI-type promoter site 34 bp upstream of the translation initiation site, which has been shown to be associated with CYP17 expression levels and thus estrogen levels (Zhang et al., 2009).

Some groups have reported an association of the $A 2$ allele (variant allele $C$ ) with increased breast cancer risk because patients with the $A 2$ allele had higher levels of circulating estrogens than those with Al alleles (Torresan et al., 2008; Zhang et al., 2009), but other studies have failed to replicate these findings (Torresan et al., 2008).

In ovarian cancer, presence of the $A 2$ variant has been associated with increased disease risk. Furthermore, the $A 2$ allele has also been associated with polycystic ovarian syndrome, a condition resulting from high androgen levels (Goodman et al., 2001).

GST enzymes have the capacity to detoxify reactive PAHs (polycyclic aromatic

Genetics and Molecular Research 16 (3): gmr16039691 
hydrocarbons) metabolites, preventing them from becoming carcinogens. These enzymes are involved in DNA protection from oxidative damage, including free radicals and metabolites generated through estrogen metabolism (Torresan et al., 2008).

Single nucleotide substitutions in GSTP1 exon 5 ( $A 313 \mathrm{G}$; Ile105Val) are in close proximity with the substrate binding site of GSTP1 and the Val variant has been demonstrated to have either lower or higher specific activity and affinity than the Ile variant, depending on the substrate (Ramalhinho et al., 2012).

Previous studies on the potential association of GSTP/polymorphisms and breast cancer have produced inconsistent results (Torresan et al., 2008; Antognelli et al., 2009) and few studies have investigated the association of this polymorphism with ovarian cancer.

Moreover, polymorphisms in short tandem repeat regions (STR), also known as microsatellites markers, have been extensively studied in tumors (Zhang_and Yu, 2007). These polymorphisms can occur due to expansion or contraction of repeat sequences giving rise to what is referred to as MSI (Zhang and Yu, 2007; Yoon et al., 2008). MSI occurs in about $90 \%$ of hereditary non-polyposis colorectal cancer (HNPCC) and has also been observed in a variety of sporadic cancers, including colon, endometrium, pancreas, and bladder (Yoon et al., 2008). In breast cancer, there is no consensus regarding the frequency of MSI. Some studies have found that MSI is not associated with this neoplasm, while others have reported frequencies that vary from 5 to $40 \%$ (Shen et al., 2000). In ovarian cancer, MSI frequency ranges from 5 to $50 \%$ (Sood et al., 2001), being the higher frequencies observed in endometrioid, mucinous and clear cell ovarian tumors (PlisieckaHałasa et al., 2008). Analysis of the highly polymorphic microsatellite loci not only provides information about MSI, but also allows for the detection of $\mathrm{LOH}$ in tumor cells (Powierska-Czarny et al., 2003).

$\mathrm{LOH}$ is thought to indicate regions harboring tumor suppressor genes and this phenomenon may also reflect random chromosomal instability. Thus, a high frequency of LOH may indicate DNA damage or instability (Tokunaga et al., 2012).

In breast and ovarian cancer, frequent $\mathrm{LOH}$ has been detected on chromosomes $3 \mathrm{p}, 6 \mathrm{q}$, $11 \mathrm{p}, 13 \mathrm{q}, 16 \mathrm{q}, 17 \mathrm{p}, 17 \mathrm{q}$, and $18 \mathrm{q}$, so that several tumor suppressor genes have been mapped to these regions (Ando et al., 2000; Plisiecka-Hałasa et al., 2008).

For all these reasons, our aim was to analyze the influence of GSTP1 and CYP17 polymorphisms in breast and ovarian cancer cases in Espírito Santo, Brazil, and their correlation with MSI/LOH in $A R, E R \beta$ and $C Y P 19$ genes, which are involved in steroid biosynthesis.

\section{MATERIAL AND METHODS}

\section{Ethics}

This study was approved by the Ethics Committee of Universidade Federal do Espírito Santo, protocol No. 02/09, including the informed consent waiver for breast and ovarian cancer cases and control group volunteers.

\section{Sample}

The study population consisted of archival formalin-fixed paraffin-embedded tissues from 107 female breast and 24 ovarian tumors, obtained from Santa Rita de Cássia Hospital

Genetics and Molecular Research 16 (3): gmr16039691 
Pathology Department, Espírito Santo State, Brazil, during years 2009 and 2010. Tumor cells were collected by a 3-mm punch in the tumor block, guided by the Pathologist. Briefly, histological slides were prepared from formalin-fixed paraffin-embedded tissues, stained with hematoxylin and eosin and allowed to air dry. Tumor tissue was marked in the hematoxylineosin stained slides by a Pathologist and slides were aligned with the block for punch extraction. Normal control tissue was obtained from the same block. General population GSTP1 and CYP17 gene polymorphism frequencies were studied from 61 healthy female blood donors at Espírito Santo Hemotherapy and Hematology Center with no previous personal or familial history of breast and ovarian cancer.

\section{DNA extraction}

Breast and ovarian cancer genomic DNA was extracted from the punch fragment by slicing and incubating at $58^{\circ} \mathrm{C}$ for five days in $0.5 \mathrm{M}$ Tris, $0.02 \mathrm{M}$ EDTA, $0.01 \mathrm{M} \mathrm{NaCl}, 1$ $\mathrm{mg} / \mathrm{mL}$ proteinase $\mathrm{K}$, and $2 \%$ SDS solution. Lysates were subsequently submitted to organic extraction with phenol-chloroform. Healthy control DNA was isolated from peripheral blood using a phenol-chloroform organic extraction.

\section{Genotyping analysis}

GSTP1 Ile-Val polymorphism was determined by PCR-RFLP (Harries et al., 1997). PCR products were digested with $1 \mathrm{U} B s m \mathrm{~A} 1$ restriction enzyme for $16 \mathrm{~h}$ at $55^{\circ} \mathrm{C}$ and visualized in silver stained $10 \%$ polyacrylamide gels. The wild-type allele was identified by the presence of a 176-bp size fragment. Variant alleles were restricted and yield two fragments of 91 and $85 \mathrm{bp}$. The presence of three fragments (176, 91 and $85 \mathrm{bp})$ indicated heterozigosity. CYP17 genotypes were also determined by PCR-RFLP (McKean-Cowdin et al., 2001). PCR product digestion was carried for $16 \mathrm{~h}$ at $37^{\circ} \mathrm{C}$ with $1 \mathrm{U}$ of MspA1I restriction enzyme and products were visualized in silver stained $7 \%$ polyacrylamide gels. The wild-type allele was identified by a 414-bp size fragment. Variant alleles produced two fragments of 290 and $124 \mathrm{bp}$. The presence of three fragments (414, 290 and $124 \mathrm{bp}$ ) indicated heterozigosity. Microsatellite markers were analyzed by PCR in simplex reactions with a final volume of 15 $\mu \mathrm{L}$ and using cycling conditions specific for each primer pair. PCR mixtures containing $1 \mathrm{X}$ PCR buffer $\left(50 \mathrm{mM} \mathrm{KCl}, 20 \mathrm{mM}\right.$ Tris-HCl, $\mathrm{pH}$ 8.4) of the Platinum ${ }^{\circledR}$ Taq DNA Polymerase (Invitrogen, Carlsbad, CA, USA), 4-10 ng genomic DNA, 0.5-1.0 $\mu \mathrm{M}$ of each primer, $0.2 \mathrm{mM}$ of each dinucleotide triphosphate, $1.5 \mathrm{mM} \mathrm{MgCl}_{2}$, and $0.6 \mathrm{U}^{\text {Platinum }}{ }^{\circledR}$ Taq DNA Polymerase (Invitrogen). Amplified fragments were resolved in 15\% polyacrylamide gels at $160 \mathrm{~V}$ for 15 $\mathrm{h}$ and visualized after silver staining.

\section{Statistical analysis}

GSTP1 and CYP17 polymorphism frequencies were analyzed using contingency tables to calculate odds ratio (OR) with a confidence interval (CI) of $95 \%$. A Fisher exact test significance value of $\mathrm{P}<0.05$ was considered, using the EpiInfo version 3.5.1 software. Hardy-Weinberg equilibrium (HWE) was estimated using the Arlequin software, version 3.11 (Excoffier et al., 2005). Quantitative variables (mean age, median and standard deviation) were analyzed by SPSS version 17.0.

Genetics and Molecular Research 16 (3): gmr16039691 


\section{RESULTS}

Histopathological characteristics of breast and ovarian tumors were summarized in Table 1. Mean and median ages for breast cancer were, respectively, 55.91 and 56.00 years (SD $=13.1$; range 28 to 89 years); as for ovarian cancer, they were 55 and 54.5 years, respectively ( $\mathrm{SD}=13.8$; range 30 to 84 years), whereas for healthy female controls, mean age was 31.8 and median age was 28 years $(\mathrm{SD}=10.8$; range 18 to 56$)$.

\begin{tabular}{|c|c|c|c|}
\hline & \multirow[t]{2}{*}{ Characteristics } & \multicolumn{2}{|c|}{ Total } \\
\hline & & $\mathrm{N}$ & $(\%)$ \\
\hline \multirow[t]{24}{*}{ Breast cancer } & Histological type & & \\
\hline & Infiltrating ductal carcinoma & 93 & (86.9) \\
\hline & Other carcinomas & 14 & $(13.1)$ \\
\hline & Histological grade ${ }^{\mathrm{a}}$ & & \\
\hline & Grade I & 5 & $(5.4)$ \\
\hline & Grade II & 57 & $(61.3)$ \\
\hline & Grade III & 31 & $(33.3)$ \\
\hline & ER status ${ }^{\mathrm{a}}$ & & \\
\hline & Negative & 16 & $(17.2)$ \\
\hline & Positive & 77 & $(82.8)$ \\
\hline & PR status $^{\mathrm{a}}$ & & \\
\hline & Negative & 25 & $(26.9)$ \\
\hline & Positive & 65 & $(69.9)$ \\
\hline & Missing & 3 & $(3.2)$ \\
\hline & HER2 status ${ }^{\mathrm{a}}$ & & \\
\hline & Negative & 88 & $(94.6)$ \\
\hline & Positive & 5 & $(5.4)$ \\
\hline & Ki67 status ${ }^{\mathrm{a}}$ & & \\
\hline & Negative & 0 & $(0.0)$ \\
\hline & Positive & 91 & $(97.8)$ \\
\hline & Missing & 2 & $(2.2)$ \\
\hline & Age at diagnosis & & \\
\hline & $\leq 45$ years & 22 & $(20.6)$ \\
\hline & $\geq 46$ years & 85 & $(79.4)$ \\
\hline \multirow[t]{9}{*}{ Ovarian cancer } & Histological type & & \\
\hline & Serous cystadenocarcinoma & 7 & $(29.2)$ \\
\hline & Mucinous cystadenocarcinoma & 1 & (4.2) \\
\hline & Other malignant & 7 & $(29.2)$ \\
\hline & Metastatic tumor & 5 & $(20.8)$ \\
\hline & Borderline tumor & 4 & $(16.7)$ \\
\hline & Age at diagnosis & & \\
\hline & $\leq 50$ years & 9 & $(37.5)$ \\
\hline & $\geq 51$ years & 15 & $(62.5)$ \\
\hline
\end{tabular}

anfiltrating ductal carcinomas only.

Regarding ethnicity, breast cancer patients were considered 22.4\% (24/107) caucasoids and $56.1 \%(60 / 107)$ mulattos, reflecting the mixed ethnicity present in this state (Perrone and Moreira, 2003). For ovarian tumor patients and healthy controls, this information was not possible to collect.

GSTP1 and CYP17 genotypic and allelic distribution in breast/ovarian cases and controls were summarized in Table 2 . We did not find any significant increase of breast and ovarian cancer risk associated with genotypes and/or alleles of these genes.

Healthy control genotypic frequencies were in HWE. Associations between breast and ovarian cancer clinicopathological characteristics with GSTP1 and CYP17 genotypic frequencies were shown in Tables 3 and 4 . We found a significant statistical association between the CYP17 A2/A2 genotype and PR-positive breast cancer $(\mathrm{P}=0.049)$. Histological

Genetics and Molecular Research 16 (3): gmr16039691 
E.V.W. dos Santos et al.

type and grade, ER and HER2 status and age at diagnosis of breast cancer did not show significant correlations with genotypes. The CYP17 A2/A2 genotype was significantly more present in the ovarian cancer age group $\leq 50$ years $(\mathrm{P}=0.027)$.

Table 2. GSTP1 and CYP17 genotypic and allelic frequencies.

\begin{tabular}{|c|c|c|c|c|c|c|c|c|}
\hline \multirow[b]{2}{*}{ GSTP1 } & \multicolumn{2}{|c|}{ Controls } & \multicolumn{3}{|c|}{ Breast cases } & \multicolumn{3}{|c|}{ Ovarian cases } \\
\hline & & & & & & & & \\
\hline Genotype & $\mathrm{N}=59$ & $\%(95 \% \mathrm{CI})$ & $\mathrm{N}=106$ & $\%(95 \% \mathrm{CI})$ & OR $(95 \% \mathrm{CI})$ & $\mathrm{N}=21$ & $\%(95 \% \mathrm{CI})$ & OR $(95 \% \mathrm{CI})$ \\
\hline Ile/Ile & 19 & $32.2(20.3-44.1)$ & 45 & $42.5(33.1-51.9)$ & 1.0 & 10 & $47.6(26.3-69.0)$ & 1.0 \\
\hline $\mathrm{Ile} / \mathrm{Val}$ & 29 & $49.2(36.4-61.9)$ & 49 & $46.2(36.7-55.7)$ & $0.71(0.33-1.53)$ & 9 & $42.9(21.6-64.1)$ & $0.59(0.18-1.95)$ \\
\hline Val/Val & 11 & $18.6(8.7-28.6)$ & 12 & $11.3(5.3-17.3)$ & $0.46(0.15-1.36)$ & 2 & $9.5(0.0-22.1)$ & $0.35(0.04-2.22)$ \\
\hline $\mathrm{Ile} / \mathrm{Val}+\mathrm{Val} / \mathrm{Val}$ & 40 & $67.8(55.9-79.7)$ & 61 & $57.5(48.1-66.9)$ & $0.64(0.31-1.32)$ & 11 & $52.4(31.0-73.8)$ & $0.52(0.17-1.62)$ \\
\hline Allele & $\mathrm{N}=118$ & & $\mathrm{~N}=212$ & & & $\mathrm{~N}=42$ & & \\
\hline Ile & 67 & $56.8(47.8-65.7)$ & 139 & $65.6(59.2-72.0)$ & 1.0 & 29 & $69.0(55.1-83.0)$ & 1.0 \\
\hline Val & 51 & $43.2(34.3-52.2)$ & 73 & $34.4(28.0-40.8)$ & $0.69(0.42-1.12)$ & 13 & $31.0(17.0-44.9)$ & $0.59(0.26-1.32)$ \\
\hline \multicolumn{9}{|l|}{ CYP17 } \\
\hline Genotype & $\mathrm{N}=61$ & & $\mathrm{~N}=69$ & & & $\mathrm{~N}=12$ & & \\
\hline$A 1 / A 1$ & 21 & $34.4(22.5-46.3)$ & 26 & $37.7(26.3-49.1)$ & 1.0 & 4 & $33.3(6.6-60.0)$ & 1.0 \\
\hline$A 1 / A 2$ & 31 & $50.8(38.3-63.3)$ & 36 & $52.2(40.4-64.0)$ & $0.94(0.41-2.12)$ & 5 & $41.7(13.8-69.6)$ & $0.85(0.17-4.35)$ \\
\hline$A 2 / A 2$ & 9 & $14.8(5.8-23.6)$ & 7 & $10.1(3.0-17.2)$ & $0.63(0.17-2.26)$ & 3 & $25.0(0.5-49.5)$ & $1.75(0.24-12.46)$ \\
\hline$A 1 / A 2+A 2 / A 2$ & 40 & $65.6(53.7-77.5)$ & 43 & $62.3(50.9-73.7)$ & $0.87(0.40-1.89)$ & 8 & $66.7(40.0-93.4)$ & $1.05(0.24-4.76)$ \\
\hline Allele & $\mathrm{N}=122$ & & $\mathrm{~N}=138$ & & & $\mathrm{~N}=24$ & & \\
\hline$A 1$ & 73 & $59.8(51.1-68.5)$ & 88 & $63.8(55.8-71.8)$ & 1.0 & 13 & $54.2(34.3-74.1)$ & 1.0 \\
\hline$A 2$ & 49 & $40.2(31.5-48.9)$ & 50 & $36.2(28.2-44.2)$ & $0.85(0.50-1.44)$ & 11 & $45.8(25.9-65.7)$ & $1.26(0.48-3.31)$ \\
\hline
\end{tabular}

Table 3. GSTP1 and CYP17 genotype distribution according to breast tumor features.

\begin{tabular}{|c|c|c|c|c|c|c|c|c|}
\hline & \multicolumn{3}{|c|}{ GSTPI } & \multirow[t]{2}{*}{$P$} & \multicolumn{3}{|c|}{ CYP17 } & \multirow[t]{2}{*}{$P$} \\
\hline & Ile/Ile & Ile/Val & Val/Val & & $A I / A I$ & $A 1 / A 2$ & $A 2 / A 2$ & \\
\hline Histological type & $\mathrm{N}(\%)$ & $\mathrm{N}(\%)$ & $\mathrm{N}(\%)$ & & $\mathrm{N}(\%)$ & $\mathrm{N}(\%)$ & $\mathrm{N}(\%)$ & \\
\hline IDC $^{\mathrm{a}}$ & $35(38.0)$ & $46(50.0)$ & $11(12.0)$ & 0.061 & $22(36.7)$ & $31(51.7)$ & $7(11.7)$ & 0.549 \\
\hline Other carcinomas & $10(71.5)$ & $3(21.5)$ & $1(7.0)$ & & $4(44.4)$ & $5(55.6)$ & $0(0.0)$ & \\
\hline \multicolumn{9}{|l|}{ Grade } \\
\hline I & $3(60.0)$ & $3(20.0)$ & $1(20.0)$ & 0.517 & $1(33.0)$ & $2(67.0)$ & $0(0.0)$ & 0.709 \\
\hline II & $20(35.0)$ & $30(53.0)$ & $7(12.0)$ & & $11(30.0)$ & $19(53.0)$ & $6(17.0)$ & \\
\hline III & $12(40.0)$ & $15(50.0)$ & $3(10.0)$ & & $10(48.0)$ & $10(48)$ & $1(4.0)$ & \\
\hline \multicolumn{9}{|l|}{ ER status } \\
\hline ER+ & $26(34.0)$ & $40(53.0)$ & $10(13.0)$ & 0.244 & $17(35.0)$ & $25(52.0)$ & $6(13.0)$ & 0.881 \\
\hline ER- & $9(56.0)$ & $6(38.0)$ & $1(6.0)$ & & $5(42.0)$ & $6(50.0)$ & $1(8.0)$ & \\
\hline \multicolumn{9}{|l|}{ PR status } \\
\hline $\mathrm{PR}+$ & $25(39.0)$ & $33(52.0)$ & $6(9.0)$ & 0.388 & 17 (41.5) & $17(41.5)$ & $7(17.0)$ & 0.049 \\
\hline PR- & $9(36.0)$ & $11(44.0)$ & $5(20.0)$ & & $5(28.0)$ & $13(72.0)$ & $0(0.0)$ & \\
\hline \multicolumn{9}{|l|}{ HER2 status } \\
\hline HER + & $2(40.0)$ & $3(60.0)$ & $0(0.0)$ & 0.690 & $3(75.0)$ & $0(0.0)$ & $1(25.0)$ & 0.101 \\
\hline HER- & $33(38.0)$ & $43(49.0)$ & $11(13.0)$ & & $19(34.0)$ & $31(55.0)$ & $6(11.0)$ & \\
\hline \multicolumn{9}{|l|}{ Age at diagnosis } \\
\hline$\leq 45$ years & $8(36.0)$ & $12(55.0)$ & $2(9.0)$ & 0.667 & $6(43.0)$ & $7(50.0)$ & $1(7.0)$ & 0.862 \\
\hline$\geq 46$ years & $37(44.0)$ & $37(44.0)$ & $10(12.0)$ & & $20(36.0)$ & $29(53.0)$ & $6(11.0)$ & \\
\hline
\end{tabular}

${ }^{\mathrm{a}}$ Infiltrating ductal carcinoma.

Table 4. GSTP1 and CYP17 genotype distribution according to ovarian tumor features.

\begin{tabular}{|c|c|c|c|c|c|c|c|c|}
\hline & \multicolumn{3}{|c|}{ GSTP1 genotypes } & \multirow[t]{2}{*}{$\mathrm{P}$} & \multicolumn{3}{|c|}{ CYP17 genotypes } & \multirow[t]{2}{*}{$\mathrm{P}$} \\
\hline & Ile/Ile & Ile/Val & $\mathrm{Val} / \mathrm{Val}$ & & $A 1 / A 1$ & $A 1 / A 2$ & $A 2 / A 2$ & \\
\hline Histologic type & $\mathrm{N}(\%)$ & $\mathrm{N}(\%)$ & $\mathrm{N}(\%)$ & & $\mathrm{N}(\%)$ & $\mathrm{N}(\%)$ & $\mathrm{N}(\%)$ & \\
\hline Ser cystadenoc ${ }^{\mathrm{a}}$ & $2(29.0)$ & $4(57.0)$ & $1(14.0)$ & 0.346 & $1(25.0)$ & $3(75.0)$ & $0(0.0)$ & 0.198 \\
\hline Muc cystadenoc $^{b}$ & $1(100.0)$ & $0(0.0)$ & $0(0.0)$ & & $0(0.0)$ & $0(0.0)$ & $0(0.0)$ & \\
\hline Others malignant & $2(40.0)$ & $2(40.0)$ & $1(20.0)$ & & $2(67.0)$ & $1(33.0)$ & $0(0.0)$ & \\
\hline Metastatic tumors & $4(100.0)$ & $0(0.0)$ & $0(0.0)$ & & $1(50.0)$ & $0(0.0)$ & $1(50.0)$ & \\
\hline Borderline tumor & $0(0.0)$ & $0(0.0)$ & $0(0.0)$ & & $0(0.0)$ & $1(33.0)$ & $2(67.0)$ & \\
\hline \multicolumn{9}{|l|}{ Age at diagnosis } \\
\hline$\leq 50$ years & $3(43.0)$ & $4(57.0)$ & $0(0.0)$ & 0.461 & $0(0.0)$ & $0(0.0)$ & $2(100.0)$ & 0.027 \\
\hline$\geq 51$ years & $7(50.0)$ & $5(36.0)$ & $2(14.0)$ & & $4(40.0)$ & $5(50.0)$ & $1(10.0)$ & \\
\hline
\end{tabular}

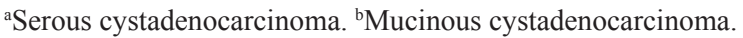

Genetics and Molecular Research 16 (3): gmr16039691 
To further investigate if GSTP1 and CYP17 might be related with breast and ovarian cancer, we analyzed their association with polymorphisms in steroid metabolism genes $A R$, $E R \beta$ and CYP19. Initially, we analyzed the combination of GSTP1 with MSI/LOH in each gene individually. Later on, we performed a combinatorial analysis of GSTP1 genotypes with MSI/ $\mathrm{LOH}$ in $A R+E R \beta, A R+C Y P 19$ or $E R \beta+C Y P 19$. Finally, we analyzed the association of GSTP1 genotypes with $\mathrm{MSI} / \mathrm{LOH}$ in $A R+E R \beta+C Y P 19$ all together. The same analyses were performed for CYP17. Associations of GSTP1 genotypes and MSI/LOH were shown in Tables 5 and 6. The Ile/Val genotype showed a correlation with $E R \beta \mathrm{MSI} / \mathrm{LOH}$ in ER-negative breast cancers $(\mathrm{P}=0.028)$, as well as the Ile/Ile genotype with $A R+C Y P 19(\mathrm{P}=0.021)$ and $A R+E R \beta+C Y P 19$ $(\mathrm{P}=0.036) \mathrm{MSI} / \mathrm{LOH}$ in PR-negative breast cancers. CYP17 Al/A1 genotype was associated with $A R+E R \beta$ and $A R+E R \beta+C Y P 19 \mathrm{MSI} / \mathrm{LOH}$ in ER- or PR-negative breast tumors $(\mathrm{P}=$ 0.039 ). No associations were detected for ovarian tumors (data not shown).

\begin{tabular}{|c|c|c|c|c|c|c|c|c|c|c|c|c|c|c|c|}
\hline \multicolumn{2}{|l|}{ GSTPI vs $A R$} & \multirow[b]{2}{*}{ ER+/ER- } & \multirow[b]{2}{*}{$\mathrm{P}$} & \multirow[b]{2}{*}{ OR $\left(95^{5} \% \mathrm{Cl}\right)$} & \multirow[b]{2}{*}{ PR+/PR- } & \multirow[b]{2}{*}{$\mathrm{P}$} & \multirow{2}{*}{ OR $(95 \% \mathrm{CI})$} & \multirow{2}{*}{\multicolumn{2}{|c|}{\begin{tabular}{c}
\multicolumn{1}{c}{ CYP17 vS $A R$} \\
Genotype $/ \mathrm{M} / \mathrm{L}^{3}$
\end{tabular}}} & \multirow[b]{2}{*}{ ER+/ER- } & \multirow[b]{2}{*}{$\mathrm{P}$} & \multirow[b]{2}{*}{ OR $(95 \% \mathrm{Cl})$} & \multirow[b]{2}{*}{ PR + /PR- } & \multirow[b]{2}{*}{$\mathrm{P}$} & \multirow{2}{*}{$\mathrm{OR}(95 \% \mathrm{Cl})$} \\
\hline $\begin{array}{l}\text { Genotype } \\
\end{array}$ & $\mathrm{M} / \mathrm{L}^{\mathrm{a}}$ & & & & & & & & & & & & & & \\
\hline He/lle & + & $0 / 2$ & 0.077 & und $^{b}$ & $\begin{array}{l}0 / 2 \\
21 / 7\end{array}$ & 0.082 & und $^{b}$ & $A I / A I$ & + & $\frac{0 / 1}{16 / 4}$ & 0.238 & und $^{b}$ & $\begin{array}{l}0 / 1 \\
16 / 4\end{array}$ & 0.238 & und $^{6}$ \\
\hline $\mathrm{He} / \mathrm{Val}$ & + & $1 / 0$ & 0.900 & $0.0(0.0-200.0)$ & $1 / 0$ & 0.790 & $\begin{array}{c}0.0(0.0-72.9) \\
\end{array}$ & $A l \mid A 2$ & + & $\frac{10 / 4}{0 / 1}$ & 0.185 & und $^{b}$ & $\begin{array}{l}0 / 1 \\
15 / 1 \\
\end{array}$ & 0.423 & und $^{b}$ \\
\hline Val/Val & + & $\frac{35 / 4}{0 / 1}$ & 0.111 & und $^{b}$ & $29 / 8$ & 0.555 & und $^{b}$ & $A 2 / A 2$ & + & $22 / 4$ & und $^{b}$ & und $^{b}$ & $\frac{15 / 10}{0 / 0}$ & und $^{b}$ & und $^{b}$ \\
\hline $\begin{array}{l}G S T P 1 \text { vs ER } \beta \\
\end{array}$ & - & $8 / 0$ & & & $4 / 4$ & & & CYPI7 & $\frac{-}{E R \beta}$ & $5 / 1$ & & & & & \\
\hline $\begin{array}{l}\text { Genotype } \\
\text { Ile/lle }\end{array}$ & $\begin{array}{ll}\mathrm{M} / \mathrm{L}^{\mathrm{a}} \\
+ \\
\end{array}$ & $\begin{array}{c}\text { ER+/ER- } \\
0 / 1 \\
\end{array}$ & $\begin{array}{c}\mathrm{P} \\
0.381 \\
\end{array}$ & $\begin{array}{c}\mathrm{OR}(95 \% \text { CI) } \\
\text { und }^{\mathrm{b}} \\
\end{array}$ & $\begin{array}{c}\text { PR+/PR- } \\
0 / 1 \\
\end{array}$ & $\begin{array}{c}\mathrm{P} \\
0.350\end{array}$ & $\begin{array}{c}\mathrm{OR}(95 \% \mathrm{Cl}) \\
\text { und }^{\mathrm{b}} \\
\end{array}$ & $\begin{array}{c}\text { Genotype } \\
A I / A I \\
\end{array}$ & $\begin{array}{c}\frac{\mathrm{M} / \mathrm{L}^{2}}{4} \\
\end{array}$ & $\begin{array}{c}\text { ER+/ER- } \\
0 / 1 \\
\end{array}$ & $\begin{array}{c}\mathrm{P} \\
0.210 \\
\end{array}$ & $\frac{\mathrm{OR}(95 \% \mathrm{Cl})}{\text { und }^{\mathrm{S}}}$ & $\begin{array}{c}\mathrm{PR}+/ \mathrm{PR}- \\
0 / 1 \\
\end{array}$ & $\begin{array}{c}\mathrm{P} \\
0.210 \\
\end{array}$ & $\begin{array}{c}\text { OR }(95 \% \mathrm{Cl}) \\
\text { und }^{6}\end{array}$ \\
\hline $\mathrm{Ile} / \mathrm{Val}$ & + & $\begin{array}{l}13 / 7 \\
13 / 2\end{array}$ & 0.028 & $\begin{array}{ll}\text { und }^{b} \\
\end{array}$ & $\begin{array}{l}13 / 6 \\
0 / 2\end{array}$ & 0.097 & und $^{b}$ & AII/A2 & + & $\begin{array}{l}0 / 1 \\
15 / 3 \\
0 / 1 \\
\end{array}$ & 0.250 & und $^{b}$ & $\begin{array}{l}0 / 1 \\
15 / 3 \\
0 / 1 \\
\end{array}$ & 0.521 & unia \\
\hline VallYal & $\because$ & $\begin{array}{l}27 / 4 \\
0 / 0\end{array}$ & und & $4 n^{b}$ & $21 / 8$ & $\mathrm{und}^{\mathrm{b}}$ & und & 42,4 & $\therefore$ & $18 / 5$ & sond & und & $011 / 11$ & $u^{b^{b}}$ & und $^{b}$ \\
\hline Talval & $t^{+}$ & $\frac{0,0}{4 / 0}$ & unde & und & $3 / 1$ & und & und & $A E A A Z$ & $+t$ & $\begin{array}{ll}0,0 \\
4 / 1\end{array}$ & und ${ }^{-}$ & Und" & $\frac{0.1}{5 / 0}$ & und & und $0^{\circ}$ \\
\hline$\frac{G S T P I \text { vs } C Y P I}{\text { Genotype }}$ & $\mathrm{M} / \mathrm{L}^{\mathrm{n}}$ & ER+/ER- & $P$ & $\mathrm{OR}\left(95^{\circ} \mathrm{Cl} \mathrm{Cl}\right.$ & $\mathrm{PR}+/ \mathrm{PR}-$ & $P$ & $\mathrm{OR}(95 \% \mathrm{Cl})$ & & $\frac{0.77 v s C}{\mathrm{M} / \mathrm{L}^{\mathrm{n}}}$ & 19 & $\mathrm{P}$ & OR $\left(95^{5} \% \mathrm{Cl}\right)$ & PR+/PR- & $\mathrm{P}$ & $\mathrm{OR}(95 \% \mathrm{Cl})$ \\
\hline He/lle & + & $1 / 0$ & 0.727 & $0.0(0.0-51.0)$ & $0 / 1$ & 0.281 & und & $A I / A I$ & + & $0 / 0$ & und $^{b}$ & und $^{b}$ & 0,0 & und $^{b}$ & und ${ }^{6}$ \\
\hline $\mathrm{Ile} / \mathrm{Val}$ & + & $\frac{23 / 9}{1 / 1}$ & 0.251 & $7.60(0.0-346.2)$ & $\begin{array}{ll}2538 \\
1 / 1\end{array}$ & 0.451 & $3.1(0.0-127.9)$ & $A 1 / A 2$ & + & $\frac{17 / 5}{1 / 0}$ & 0.800 & $0.0(0.0-81.1)$ & $\frac{1775}{0 / 1}$ & 0.448 & und $^{b}$ \\
\hline $\begin{array}{l}\text { Val/Val } \\
\end{array}$ & + & $\frac{38 / 5}{0 / 1}$ & 0.111 & und $^{b}$ & $\begin{array}{l}31 / 10 \\
0 / 1 \\
\end{array}$ & 0.555 & und $^{b}$ & $A 22 / A 2$ & + & $\begin{array}{l}23 / 6 \\
1 / 0 \\
\end{array}$ & 0.857 & $0.0(0.0-636.3)$ & $\frac{16 / 12}{1 / 0}$ & und $^{b}$ & und $^{b}$ \\
\hline & - & $8 / 0$ & & & $4 / 4$ & & & & - & $5 / 1$ & & & $6 / 0$ & & \\
\hline
\end{tabular}

${ }^{\mathrm{a} M S I+L O H . ~}{ }^{\mathrm{b}}$ Undetermined.

Table 6. GST1 and $C Y P 17$ association with $A R, E R \beta$ and $C Y P 19$, stratified by ER/PR status in breast cancer.

\begin{tabular}{|c|c|c|c|c|c|c|c|c|c|c|c|c|c|c|c|}
\hline \multicolumn{3}{|c|}{ GSTPI v $A R+E R \beta$} & & & & & & \multicolumn{3}{|c|}{ CYP17 vs $A R+E R \beta$} & \multirow{3}{*}{$\mathrm{P}$} & \multirow[b]{2}{*}{$\mathrm{OR}(95 \% \mathrm{Cl})$} & \multirow[b]{2}{*}{$\mathrm{PR}+/ \mathrm{PR}-$} & \multirow{3}{*}{$\begin{array}{c}\mathrm{P} \\
0.039 \\
\end{array}$} & \multirow{3}{*}{$\frac{\mathrm{OR}(95 \% \mathrm{CI})}{\mathrm{und}^{\mathrm{C}}}$} \\
\hline Genotype & & ER+/ER- & $\mathrm{P}$ & $\mathrm{OR}(95 \% \mathrm{Cl})$ & $\mathrm{PR}+$ PR- & $\mathrm{P}$ & $\mathrm{OR}(95 \% \mathrm{CI})$ & Genotype & $\mathrm{M} / \mathrm{L}^{\mathrm{a}}$ & ER+/ER- & & & & & \\
\hline He/lle & + & $0 / 2$ & 0.147 & und $^{b}$ & $0 / 2$ & 0.122 & und $^{b}$ & $A l / A I$ & & $0 / 2$ & & und $^{\mathrm{b}}$ & $0 / 2$ & & \\
\hline $\mathrm{He} / \mathrm{Val}$ & + & $12 / 6$ & 0.060 & $167(0.8-6867)$ & $\frac{12 / 5}{1 / 2}$ & 0.176 & $67(04-228.1)$ & $A l / 42^{2}$ & + & $\frac{14 / 2}{0 / 1}$ & 0.238 & und $\mathrm{d}^{b}$ & $\frac{14 / 2}{0,1}$ & 0.500 & $\mathrm{nin}^{\mathrm{b}}$ \\
\hline & & $25 / 3$ & & & $20 / 6$ & & & & & $\frac{0 / 1}{16 / 4}$ & & & $\frac{0 / 1}{10 / 9}$ & & \\
\hline Val/Val & + & $0 / 1$ & 0.200 & und $^{b}$ & $0 / 1$ & 0.400 & und $^{b}$ & $\overline{A 2 / A 2}$ & + & $0 / 0$ & und $^{b}$ & und $^{b}$ & $0 / 0$ & und $^{b}$ & und $^{b}$ \\
\hline CSTP & $\therefore$ & $4 / 0$ & & & $3 / 1$ & & & & $u^{R+}$ & $4 / 1$ & & & $5 / 0$ & & \\
\hline 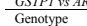 & $\frac{+C P T 9}{M / L^{4}}$ & ER+/ER- & $\mathrm{P}$ & OR $(95 \% \mathrm{Cl})$ & $\begin{array}{l}\mathrm{PR}+\mathrm{PRR}- \\
\end{array}$ & $P$ & $\mathrm{OR}(95 \% \mathrm{CI})$ & $\frac{\text { CYP }}{\text { Genotype }}$ & $\frac{\mathrm{M} / \mathrm{L}^{\mathrm{u}}}{\mathrm{M}}$ & $\frac{Y P P}{\mathrm{ER}+/ \mathrm{ER}-}$ & $P$ & $\mathrm{OR}(95 \% \mathrm{CI})$ & PR+/PR- & P & $\mathrm{OR}\left(95^{\circ} \mathrm{CD}\right)$ \\
\hline Ilenlle & $\frac{10}{+}+$ & $1 / 2$ & 0.195 & $6.0(0.3-199.8)$ & $0 / 3$ & 0.021 & und $^{\mathrm{b}}$ & $\frac{A l / A I}{A}$ & + & $0 / 1$ & 0.238 & und $^{5}$ & $\frac{0 / 1}{0 / 1}$ & 0.238 & $\frac{u^{2}(3)}{u^{d}}$ \\
\hline lleolol & - & $21 / 7$ & 0.337 & $40(00898$ & $21 / 6$ & 0567 & $160(00-398)$ & $41 / 42$ & & $16 / 4$ & & & $16 / 4$ & & \\
\hline Heval & $\frac{7}{-1}$ & $33 / 4$ & 0.391 & $4.1(0.0-89.8)$ & $27 / 18$ & 0.567 & $1.69(0.0-29.8)$ & $A T / A 2$ & + & $\frac{1 / 1}{21 / 4}$ & 0.342 & $3.3(0.0-257.8)$ & $\frac{0,2}{15 / 9}$ & 0.169 & und" \\
\hline ValVal & + & $0 / 1$ & 0.111 & und $^{b}$ & $0 / 1$ & 0.555 & und $^{b}$ & $A 2 / A 2$ & + & $1 / 0$ & 0.833 & $0.0(0.0-527.4)$ & $1 / 0$ & und $^{b}$ & und $^{b}$ \\
\hline & & $8 / 0$ & & & $4 / 4$ & & & & & $4 / 1$ & & & $5 / 0$ & & \\
\hline GSTPI v ES & $3+C Y P I$ & & & & & & & CYPI & $S E R \beta+$ & & & & & & \\
\hline Genotype & $\mathrm{M} / \mathrm{L}^{\mathrm{a}}$ & ER+/ER- & $\mathrm{P}$ & $\mathrm{OR}(95 \% \mathrm{CI})$ & $\mathrm{PR}+/ \mathrm{PR}-$ & $\mathrm{P}$ & $\mathrm{OR}(95 \% \mathrm{Cl})$ & Genotype & $\mathrm{M} / \mathrm{L}^{\mathrm{a}}$ & ER+/ER- & $\mathrm{P}$ & $\mathrm{OR}\left(95^{\circ} \% \mathrm{CI}\right)$ & $\mathrm{PR}+/ \mathrm{PR}-$ & $\mathrm{P}$ & $\mathrm{OR}\left(95^{5} \% \mathrm{Cl}\right)$ \\
\hline Ile/lle & + & $\begin{array}{l}/ 1 \\
117\end{array}$ & 0.653 & $1.6(0.0-71.2)$ & $0,0 / 2$ & 0.123 & und $^{\mathrm{b}}$ & Al/AI & + & $\begin{array}{l}0 / 1 \\
1 / 3\end{array}$ & 0.210 & und $^{6}$ & $\begin{array}{l}0 / 1 \\
1 / 3\end{array}$ & 0.210 & \\
\hline Ile/Val & + & $\frac{11 / 2}{1 / 2}$ & 0.083 & $12.5(0.6-465.2)$ & $1 / 2$ & 0.251 & $4.8(0.3-156.0)$ & $A 1 / A 2$ & + & $\frac{15 / 3}{1 / 1}$ & 0.462 & $3.2(0.0-150.4)$ & $\frac{15 / 3}{0 / 2}$ & 0.286 & und $^{b}$ \\
\hline & & $25 / 4$ & & & $19 / 8$ & & & & - & $16 / 5$ & & & $10 / 10$ & & \\
\hline $\begin{array}{l}\text { ValVal } \\
\end{array}$ & + & $0 / 1$ & 0.200 & und $^{b}$ & $0 / 1$ & 0.400 & und $^{b}$ & $A 2 / A 2$ & + & $1 / 0$ & 0.800 & $0.0(0.0-418.5)$ & $1 / 0$ & und $^{b}$ & und $^{b}$ \\
\hline GSTPI VS $A R$ & $+F R B+c$ & $4 / 0$ & & & $3 / 1$ & & & & $7 v S A R$ & $\frac{3 / 1}{E R B+C Y P I S}$ & & & $4 / 0$ & & \\
\hline Genotype & $\mathrm{M} / \mathrm{L}^{\mathrm{a}}$ & ER+/ER- & $\mathrm{P}$ & $\mathrm{OR}(95 \% \mathrm{CI})$ & $\mathrm{PR}+/ \mathrm{PR}-$ & $\mathrm{P}$ & $\mathrm{OR}(95 \% \mathrm{Cl})$ & $\frac{4}{\text { Genotype }}$ & $\mathrm{M} / \mathrm{L}^{\mathrm{a}}$ & $\frac{E R P+C T T S}{E R+/ E R}$ & $\mathrm{P}$ & OR $(95 \% \mathrm{Cl})$ & $P R+/ P R-$ & $\mathrm{P}$ & $\mathrm{OR}\left(95^{\circ} \% \mathrm{CD}\right.$ \\
\hline Ile/lle & + & $1 / 2$ & 0.344 & $3.7(0.2-129.9)$ & $0 / 3$ & 0.036 & und $^{\mathrm{b}}$ & Al/AI & + & & 0.039 & und $^{6}$ & $0 / 2$ & 0.039 & und ${ }^{\circ}$ \\
\hline 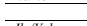 & - & $11 / 6$ & - & $77(105400)$ & $12 / 4$ & 资 & $30(0) 213$ & 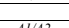 & - & $14 / 2$ & 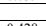 & $38(00187 ?$ & $14 / 2$ & 0 & ( \\
\hline Herfal & + & $2 / 2 / 3$ & 0.119 & $7.7(0.5-143.0)$ & $\frac{2 / 2}{18 / 6}$ & 0.318 & $3.0(0.2-41.3)$ & $A T / A 2$ & + & $\frac{1 / 1}{15 / 4}$ & 0.428 & $3.8(0.0-187.2)$ & $\frac{0 / 2}{10 / 8}$ & 0.237 & und $^{3}$ \\
\hline Val/Val & + & $\frac{23 / 1}{0 / 1}$ & 0.200 & und $^{b}$ & $\frac{18 / 6}{0 / 1}$ & 0.400 & und $^{b}$ & $A 2 / A 2$ & + & $\frac{1514}{1 / 0}$ & 0.800 & $0.0(0.0-418.5)$ & $\frac{1.18}{1 / 0}$ & und $^{b}$ & und $^{b}$ \\
\hline & & $4 / 0$ & & & $3 / 1$ & & & & & $3 / 1$ & & & $4 / 0$ & & \\
\hline
\end{tabular}

${ }^{\mathrm{a}} \mathrm{MSI}+\mathrm{LOH} .{ }^{\mathrm{b}}$ Undetermined. 


\section{DISCUSSION}

There is substantial evidence that steroid hormones play an important role in the etiology of breast and ovarian cancer (Lurie et al., 2009; Dumas and Diorio, 2011). Therefore, there has been recent interest in steroid metabolism genes, because the activity of these gene products may affect long-term estrogen levels and its potentially carcinogenic metabolites, influencing breast and ovarian cancer risk (Garner et al., 2002).

In most studies, estrogen biosynthesis and metabolism gene polymorphisms are considered separately (Ramalhinho et al., 2012), finding a small effect of low-penetrance breast and ovarian cancer risk gene polymorphisms (Zhang et al., 2009). In our study, we have analyzed SNPs in two low-penetrance genes and their association with MSI/LOH in steroid metabolism genes. Specific associations between gene polymorphisms and MSI/LOH could result in high-risk profiles, by influencing lifetime estrogen levels and therefore breast and ovarian cancer risk (Ramalhinho et al., 2012).

We performed a case-control study of Brazilian women to investigate the association of GSTP1 and CYP17 gene polymorphisms with breast and ovarian cancer, as well as with $\mathrm{MSI} / \mathrm{LOH}$ in three genes implicated in steroid metabolism $(A R, E R \beta$ and $C Y P 19)$. When tested alone, the GSTP1 gene showed no significant association with breast and ovarian cancer risk. Most previous studies on the potential association of GSTP1 polymorphisms and breast and ovarian cancer risk produced inconsistent results. Spurdle et al. (2001), Delort et al. (2008) and Ramalhinho et al. (2011) showed results similar to ours, whereas an association between GSTP1 genotypes and breast cancer risk was observed by Torresan et al. (2008) in Euro-descendent women from Southern Brazil and by Antognelli et al. (2009), which found that the frequency of the $\mathrm{Val}$ allele was significantly lower in the breast cancer population. Inconsistencies may be partly due to differences in populations and their exposures to different environmental risk factors (Torresan et al., 2008).

A combinatorial analysis of GSTP1 genotypes and MSI/LOH in $A R, E R \beta$ or $C Y P 19$ enabled us to detect that the Ile/Ile genotype is related to MSI/LOH in $A R+C Y P 19$ or $A R+E R \beta+C Y P 19$ in PR-negative breast tumors (Table 6), suggesting that this genotype when combined with MSI/LOH may be associated with poor prognosis of breast cancer.

According to Ramalhinho et al. (2011), the GSTP1 Ile/Ile genotype increases breast cancer risk when associated with GSTM1/GSTT1 null genotypes, suggesting that the Val allele tends to act as a protective, rather than a risk factor.

Regarding CYP17 polymorphism distribution in breast and ovarian tumors, there are also controversial evidences. Early reports suggested an association of CYP17 A2 with an increased breast cancer risk; however, subsequent studies failed to confirm this association (Garner et al., 2002; Miyoshi and Noguchi, 2003). As for ovarian cancer risk, Goodman et al. (2001) and Spurdle et al. (2001) reported no evidence for an association between CYP17 polymorphisms and ovarian cancer risk, while Garner et al. (2002) noted an increased risk for ovarian cancer in $A 2$ variant women $>50$ years.

Our results did not show a significant association between $C Y P 17$ polymorphisms and breast and ovarian cancer risk. However, when we analyzed the relation between CYP17 genotypes and histopatological characteristics of breast tumors, a statistically significant frequency of the $A 2 / A 2$ genotype in patients with PR-positive breast cancers was found (Table $3)$. For ovarian cancer, a significant frequency of the $A 2 / A 2$ genotype was observed in the age at diagnostic group $\leq 50$ years (Table 4 ).

Genetics and Molecular Research 16 (3): gmr16039691 
Because the $A 2$ variant allele creates an additional putative Sp- 1 binding site (CCACC) in the $C Y P 17$ promoter region, it is speculated that the $C$ allele enhances gene transcription, leading to increased estrogen synthesis in breast and ovarian tumors (Garner et al., 2002; Zhang et al., 2009).

A combinatorial analysis of $C Y P 17$ genotypes with MSI/LOH in $A R, E R \beta$ or $C Y P 19$ genes allowed for the detection of a correlation between the $A 1 / A 1$ genotype and a higher frequency of $\mathrm{MSI} / \mathrm{LOH}$ in $A R+E R \beta$ or $A R+E R \beta+C Y P 19$ in ER-negative and PR-negative tumors (Table 6). Similarly, the CYP17 wild-type genotype when combined with MSI/LOH was associated with poor prognosis of breast cancer.

$A R, E R \beta$ and CYP19 genes participate in the androgen hormone pathway. The androgen receptor $(A R)$ has a polymorphic polyglutamine repeat (CAG repeat) in the aminoterminal domain while the $E R \beta$ gene contains a polymorphic dinucleotide CA repeat in the non-coding 3'-portion of the gene. Polymorphic repeats appear to influence function and alter androgen serum levels in premenopausal women (Westberg et al., 2001), because sex hormone receptors $(A R$ and $E R \beta)$ mediate hormone response at breast tissue level, having a possible pathological role in breast cancer development (Anghel et al., 2006). CYP19 (aromatase) is involved in the last stage of androgen to estrogen conversion (androstenedione into estrone and testosterone into estradiol) (Miyoshi and Noguchi, 2003; Zhang et al., 2009) and its activity helps determines local estrogen levels. MSI in intron 5 polymorphic tetranucleotide repeat has been observed in human breast, ovary, soft tissue and brain carcinomas. This aromatase gene region may be involved in splice site determination (Kristensen and Borresen-Dale, 2000; Huber et al., 2002).

To our knowledge, this is the first study to analyze the potential role of GSTP1 and $C Y P 17$ genotypes in combination with $A R, E R \beta$ and $C Y P 19 \mathrm{MSI} / \mathrm{LOH}$ in Brazilian women with breast or ovarian tumors. We observed that wild-type GSTP1 and CYP17 genotypes when combined with MSI/LOH in steroid metabolism genes is associated with ER-negative or PRnegative breast cancers. These results support the hypothesis that estrogen metabolism genes can be helpful in the characterization of breast cancer prognosis (Ramalhinho et al., 2012).

\section{Conflicts of interest}

The authors declare no conflicts of interest.

\section{ACKNOWLEDGMENTS}

Research partly supported by Fibria Celulose. E.V.W. dos Santos was supported by a CNPq doctorate scholarship. L.N.R. Alves was supported by a CNPq scholarship.

\section{REFERENCES}

Ando Y, Iwase H, Ichihara S, Toyoshima S, et al. (2000). Loss of heterozygosity and microsatellite instability in ductal carcinoma in situ of the breast. Cancer Lett. 156: 207-214. https://doi.org/10.1016/S0304-3835(00)00467-5

Anghel A, Raica M, Marian C, Ursoniu S, et al. (2006). Combined profile of the tandem repeats CAG, TA and CA of the androgen and estrogen receptor genes in breast cancer. J. Cancer Res. Clin. Oncol. 132: 727-733. https://doi. org/10.1007/s00432-006-0121-9

Antognelli C, Del Buono C, Ludovini V, Gori S, et al. (2009). CYP17, GSTP1, PON1 and GLO1 gene polymorphisms as risk factors for breast cancer: an Italian case-control study. BMC Cancer 9: 115 https://doi.org/10.1186/1471-2407-9-115.

Genetics and Molecular Research 16 (3): gmr16039691 
Delort L, Chalabi N, Satih S, Rabiau N, et al. (2008). Association between genetic polymorphisms and ovarian cancer risk. Anticancer Res. 28 (5B): 3079-3081.

Dumas I and Diorio C (2011). Estrogen pathway polymorphisms and mammographic density. Anticancer Res. 31: 4369 4386.

Excoffier, Laval LG and Schneider S (2005). Arlequin version 3.0: an integrated software package for population genetics data analysis. Evol. Bioinform. Online 1: 47-50.

Garner EI, Stokes EE, Berkowitz RS, Mok SC, et al. (2002). Polymorphisms of the estrogen-metabolizing genes CYP17 and catechol-O-methyltransferase and risk of epithelial ovarian cancer. Cancer Res. 62: 3058-3062.

Goodman MT, McDuffie K, Guo C, Terada K, et al. (2001). CYP17 genotype and ovarian cancer: a null case-control study. Cancer Epidemiol. Biomarkers Prev. 10: 563-564.

Harries LW, Stubbins MJ, Forman D, Howard GC, et al. (1997). Identification of genetic polymorphisms at the glutathione S-transferase Pi locus and association with susceptibility to bladder, testicular and prostate cancer. Carcinogenesis 18: 641-644. https://doi.org/10.1093/carcin/18.4.641

Huan Z, Nakayama K, Nakayama N, Ishibashi M, et al. (2008). Genetic classification of ovarian carcinoma based on microsatellite analysis: relationship to clinicopathological features and patient survival. Oncol. Rep. 19: 775-781.

Huber JC, Schneeberger C and Tempfer CB (2002). Genetic modelling of the estrogen metabolism as a risk factor of hormone-dependent disorders. Maturitas 42: 1-12. https://doi.org/10.1016/S0378-5122(02)00021-X

Imyanitov EM and Hanson KP (2004). Mechanisms of breast cancer. Drug Discov. Today Dis. Mech. 1: 235-245. https:// doi.org/10.1016/j.ddmec.2004.09.002

Kristensen VN and Borresen-Dale AL (2000). Molecular epidemiology of breast cancer: genetic variation in steroid hormone metabolism. Mutat. Res. 462: 323-333. https://doi.org/10.1016/S1383-5742(00)00018-1

Lurie G, Wilkens LR, Thompson PJ, McDuffie KE, et al. (2009). Genetic polymorphisms in the estrogen receptor beta (ESR2) gene and the risk of epithelial ovarian carcinoma. Cancer Causes Control 20: 47-55. https://doi.org/10.1007/ $\underline{\text { s10552-008-9216-8 }}$

Lynch HT, Casey MJ, Snyder CL, Bewtra C, et al. (2009). Hereditary ovarian carcinoma: heterogeneity, molecular genetics, pathology, and management. Mol. Oncol. 3: 97-137. https://doi.org/10.1016/j.molonc.2009.02.004

McKean-Cowdin R, Feigelson HS, Pike MC, Coetzee GA, et al. (2001). Risk of endometrial cancer and estrogen replacement therapy history by CYP17 genotype. Cancer Res. 61: 848-849.

Miyoshi Y and Noguchi S (2003). Polymorphisms of estrogen synthesizing and metabolizing genes and breast cancer risk in Japanese women. Biomed. Pharmacother. 57: 471-481. https://doi.org/10.1016/j.biopha.2003.09.008

Perrone A and Moreira THL (2003). História e Geografia do Espírito Santo. 1st edn. Gráfica Sodré, Vitória.

Plisiecka-Hałasa J, Dansonka-Mieszkowska A, Kraszewska E, Dańska-Bidzińska A, et al. (2008). Loss of heterozygosity, microsatellite instability and TP53 gene status in ovarian carcinomas. Anticancer Res. 28 (2A): 989-996.

Powierska-Czarny J, Miścicka-Sliwka D, Czarny J, Grzybowski T, et al. (2003). Analysis of microsatellite instability and loss of heterozygosity in breast cancer with the use of a well characterized multiplex system. Acta Biochim. Pol. 50: 1195-1203.

Ramalhinho AC, Fonseca-Moutinho JA and Breitenfeld L (2011). Glutathione S-transferase M1, T1, and P1 genotypes and breast cancer risk: a study in a Portuguese population. Mol. Cell. Biochem. 355: 265-271. https://doi.org/10.1007/ s11010-011-0863-9

Ramalhinho AC, Fonseca-Moutinho JA and Breitenfeld Granadeiro LA (2012). Positive association of polymorphisms in estrogen biosynthesis gene, CYP19A1, and metabolism, GST, in breast cancer susceptibility. DNA Cell Biol. 31: 1100-1106. https://doi.org/10.1089/dna.2011.1538

Shen KL, Yang LS, Hsieh HF, Chen CJ, et al. (2000). Microsatellite alterations on human chromosome 11 in in situ and invasive breast cancer: a microdissection microsatellite analysis and correlation with p53, ER (estrogen receptor), and PR (progesterone receptor) protein immunoreactivity. J. Surg. Oncol. 74: 100-107. https://doi.org/10.1002/10969098(200006)74:2<100::AID-JSO5>3.0.CO;2-O

Sood AK, Holmes R, Hendrix MJ and Buller RE (2001). Application of the National Cancer Institute international criteria for determination of microsatellite instability in ovarian cancer. Cancer Res. 61: 4371-4374.

Spurdle AB, Webb PM, Purdie DM, Chen X, et al. (2001). Polymorphisms at the glutathione S-transferase GSTM1, GSTT1 and GSTP1 loci: risk of ovarian cancer by histological subtype. Carcinogenesis 22: 67-72. https://doi. org/10.1093/carcin/22.1.67

Tokunaga E, Okada S, Yamashita N, Akiyoshi S, et al. (2012). High incidence and frequency of LOH are associated with aggressive features of high-grade HER2 and triple-negative breast cancers. Breast Cancer 19: 161-169. https://doi. org $/ 10.1007 / \mathrm{s} 12282-010-0232-7$

Torresan C, Oliveira MM, Torrezan GT, de Oliveira SF, et al. (2008). Genetic polymorphisms in oestrogen metabolic

Genetics and Molecular Research 16 (3): gmr16039691 
pathway and breast cancer: a positive association with combined CYP/GST genotypes. Clin. Exp. Med. 8: 65-71. https://doi.org/10.1007/s10238-008-0159-x

Westberg L, Baghaei F, Rosmond R, Hellstrand M, et al. (2001). Polymorphisms of the androgen receptor gene and the estrogen receptor beta gene are associated with androgen levels in women. J. Clin. Endocrinol. Metab. 86: 25622568.

Yoon BS, Kim YT, Kim JH, Kim SW, et al. (2008). Clinical significance of microsatellite instability in sporadic epithelial ovarian tumors. Yonsei Med. J. 49: 272-278. https://doi.org/10.3349/ymj.2008.49.2.272

Zhang L, Gu L, Qian B, Hao X, et al. (2009). Association of genetic polymorphisms of ER-alpha and the estradiolsynthesizing enzyme genes CYP17 and CYP19 with breast cancer risk in Chinese women. Breast Cancer Res. Treat. 114: 327-338. https://doi.org/10.1007/s10549-008-9998-0

Zhang W and Yu YY (2007). Polymorphisms of short tandem repeat of genes and breast cancer susceptibility. Eur. J. Surg. Oncol. 33: 529-534. https://doi.org/10.1016/j.ejso.2006.11.027

Genetics and Molecular Research 16 (3): gmr16039691 